\title{
Philosophiques
}

\section{Stéphane Chauvier, Justice internationale et solidarité, Nîmes, Éditions Jaqueclines Chambon, 1999, 155 pages.}

\section{Martin Leblanc}

Volume 30, numéro 2, automne 2003

URI : https://id.erudit.org/iderudit/008657ar

DOI : https://doi.org/10.7202/008657ar

Aller au sommaire du numéro

Éditeur(s)

Société de philosophie du Québec

ISSN

0316-2923 (imprimé)

1492-1391 (numérique)

Découvrir la revue

Citer ce compte rendu

Leblanc, M. (2003). Compte rendu de [Stéphane Chauvier, Justice internationale et solidarité, Nîmes, Éditions Jaqueclines Chambon, 1999, 155 pages.]

Philosophiques, 30(2), 458-461. https://doi.org/10.7202/008657ar d'utilisation que vous pouvez consulter en ligne.

https://apropos.erudit.org/fr/usagers/politique-dutilisation/ 


\section{Stéphane Chauvier, Justice internationale et solidarité, Nîmes, Éditions Jaqueclines Chambon, 1999, 155 pages.}

Justice internationale et solidarité est l'un des rares ouvrages de langue française qui s'inscrit parfaitement dans le débat «anglo-saxon» sur les théories contemporaines de la justice sociale internationale. Dans ce livre, Stéphane Chauvier cherche à repenser les présupposés contractualistes selon une perspective cosmopolite. Selon lui, les théories du contrat social auraient fait fausse route en essayant de résoudre d'abord le problème de la justice "domestique» puis ensuite d'aborder celui du droit des gens. Ce type de contrat mènerait à une théorie du "bon voisinage » entre les États, mais non à une véritable théorie de la justice. Il s'oppose donc au droit des gens de John Rawls, qu'il considère comme une théorie de la "courtoisie internationale". Sa thèse est la suivante: le contrat "originel» doit être global et il doit précéder l'établissement des conventions régionales. La justice interpersonnelle doit avoir préséance sur la justice internationale, pour ainsi dire. Les contractants dans la position originelle doivent donc représenter des individus et non des États.

D'entrée de jeu, Chauvier signale dans son introduction que la tradition philosophique n'a pas accordé beaucoup d'importance aux questions de la justice hors frontières. De la République de Platon jusqu'à la Théorie de la justice de Rawls, l'attention des philosophes politiques se serait ainsi presque exclusivement portée sur les problèmes de justice interne (la répartition des pouvoirs, la distribution de la richesse, les droits entre membres d'un même État). La "cité » a toujours été l'espace par défaut dans lequel le problème de la justice devait être traité. Mais pourquoi devrait-il en être ainsi? Pourquoi ne pas penser la justice globalement?

L'essentiel du premier chapitre de Justice internationale et solidarité consiste à démontrer que la tradition philosophique du droit des gens n'a produit qu'une résolution théorique du problème de la guerre entre les États, non une théorie de la justice proprement dite. Selon Chauvier, parce qu'elle ne s'est pas souciée des inégalités de dotation entre les individus se situant dans différents États, la tradition philosophique n'a pas produit une théorie de la justice proprement dite, mais une théorie de la civilité ou de la courtoisie entre les États. «Or, affirme Chauvier, entre des bandes de brigands, il peut y avoir de telles règles de civilité. Des groupes de brigands peuvent s'entendre pour se partager les revenus d'un secteur. Même dans une société qui se conformerait aux principes du Droit des Gens, la justice pourrait donc rester un vaste brigandage» (p. 21).

Non seulement la théorie traditionnelle du droit des gens n'est pas une théorie de la justice, mais elle ne permet pas d'aborder les principaux problèmes internationaux de notre temps: le problème du droit international des personnes et de la place de l'individu dans la société internationale, le problème du droit des générations futures et des servitudes collectives, le problème enfin de la disproportion des dotations économiques des États, de leur inégale dotation en ressources naturelles, humaines et capitalistiques de production (p. 33). Pour Chauvier, le principe classique de la pleine souveraineté des États qui est à la base de la théorie du droit des gens bloque toute réflexion à propos de ces problèmes pourtant cruciaux.

Au second chapitre, Chauvier précise que les exigences d'une théorie de la justice cosmopolite ne peuvent pas se réaliser à l'intérieur du cadre adopté par 
Rawls dans son droit des gens. Il ne s'agirait donc pas simplement d'adapter la théorie, il faudrait la repenser totalement. Chauvier pense comme Thomas Pogge que la justice cosmopolite doit prendre en compte les intérêts des individus, mais il ne pense pas que cela puisse se faire dans le cadre adopté par Rawls (Thomas Pogge, "An Egalitarian Law of Peoples », Philosophy and Public Affairs, été 1994, vol. 23, $\mathrm{n}^{\circ} 3$, p. 195-224.). Le problème du droit international des personnes, comme bien d'autres problèmes internationaux de notre temps, ne pourrait pas figurer parmi les objets d'une délibération entre représentants d'États réunis en position originelle. Les considérations relatives à la formulation de principes de solidarité, de coopération et de redistribution des richesses ne concerneraient tout simplement pas la société des États. "La délibération [entre États] a pour objet de créer un environnement international qui permette à chaque État de préserver son bon ordre interne et elle présuppose donc nécessairement que le représentant de chaque État sait que son État est bien ordonné » (p. 59). Par conséquent, "cela n'a pas de sens de supposer qu'un État qui se soucie d'édifier une société internationale bien ordonnée puisse désirer la puissance et la richesse et qu'il puisse faire l'hypothèse qu'il est mal ordonné » (p. 60). Chauvier conclut donc qu'il faut abandonner le point de vue de l'État si l'on veut dépasser la conception traditionnelle du droit des gens.

Aux troisième, quatrième et cinquième chapitres, Chauvier explicite son idée de position originelle globale. Cette position ferait référence à une situation dans laquelle des individus plutôt que des représentants d'États se réuniraient pour établir les bases de la société internationale. Dans cette situation, Chauvier est d'avis que les personnes rationnelles et désireuses de maximiser leurs avantages exigeront davantage qu'un simple code de bon voisinage. Ils s'accorderaient sur un principe de collaboration sociale. Toutefois, les partenaires ne seraient pas amenés à former les uns avec les autres un État, mais à se répartir en États distincts. L'auteur estime que les contractants, mis au fait du pluralisme, choisiraient de se séparer parce qu'ils jugeraient, pour différentes considérations, que la séparation serait dans l'intérêt de tous et de chacun (p. 75-100). Sans doute pour des raisons d' "efficacité », pour respecter les différentes cultures, les différentes traditions et les appartenances nationales, les contractants jugeraient préférable de ne pas se constituer en un État mondial. Cette séparation «cosmopolite» serait dans l'intérêt de chacun, dans l'intérêt même de l'humanité.

Chauvier est d'avis que les principes de la justice internationale doivent être compris comme des principes d'une équitable séparation politique des gens: «la séparation ne peut être complètement voulue que si elle est équitable, et elle ne peut être équitable si certaines conditions ne sont pas imposées à la séparation" (p. 86). Il est conscient que la géographie de la Terre, le climat mais aussi les différentes cultures et traditions peuvent influencer la richesse des nations. Or, parce que la séparation politique peut placer les personnes dans des situations qui sont non seulement différentes mais inégales, «[les contractants] chercheront, en tenant compte de la perspective de ces inégales dotations, à définir une société non point tant de coopération mutuelle que de correction continuelle de ces désajustements originels» (p. 95). La séparation politique des hommes devra être considérée comme un processus continu et non pas comme un événement fixe et défini dans le temps. Vu sous cette angle, il pense donc qu' «il n'y a pas de place perdante en soi ", parce que " ceux qui gagnent s'efforcent toujours de permettre aux autres de gagner aussi »(p. 100). 
Aux sixième et septième chapitres, Chauvier soutient que sa théorie de la justice cosmopolite doit être comprise comme une théorie de la justice "sédentaire» et non pas comme une théorie de la justice "nomadique». Selon lui, les contractants réunis en position originelle globale ne pourraient se satisfaire d'un monde dans lequel la justice cosmopolite se réduirait au droit de changer de pays. Elle devrait plutôt se concevoir comme un ensemble de conditions offrant aux défavorisés une autre possibilité que l'émigration forcée. Si la solution nomadique a l'intérêt d'être des plus économique, elle ne respecterait pas cette tendance naturelle générale des gens à vouloir vivre là où ils sont nés. Il s'avérerait donc plus plausible que les contractants s'accordent sur une solution plus "sédentaire ", solution qui serait davantage compatible avec la préservation du bon ordre interne de l'État.

D'après Chauvier, il est clair que les contractants réunis dans la position originelle globale accepteraient les principes traditionnels du droit des gens (reconnaissance de l'égalité juridique des États, principe du respect des traités et engagements, droit à l'autodéfense, etc.), mais ils exigeraient aussi davantage. En effet, écrit-il, l'intérêt du point vue cosmopolite vient du fait qu'il permet d'aller audelà de ces principes traditionnels: "Si l'on se met à raisonner non plus en représentant d'un État quelconque, mais en citoyen d'un État quelconque, nous sommes nécessairement conduits à intégrer au corps de la justice internationale bien d'autres principes que ceux du respect mutuel de la tradition du Droit des Gens » (p. 119120). En plus des principes traditionnels du droit des gens, les contractants s'accorderaient selon Chauvier sur un principe de "socialisation des avantages» (p. 125). Ce principe se traduirait par: (a) un devoir d'ingérence des États et des personnes les mieux nantis partout où l'une des conceptions de la justice admises dans la société des États se trouve violée et (b) une clause d' "accessibilité des ressources" .

Justice internationale et solidarité est un ouvrage remarquable. Les théoriciens qui suivent les débats contemporains sur la justice internationale, la philosophie des relations internationales et du droit international ne pourront pas éviter ce livre. Il faut saluer la tentative originale de l'auteur visant à présenter le contrat social international selon une perspective franchement cosmopolite sans pour autant évacuer les problèmes liés au pluralisme des valeurs. Bien qu'il le fasse à certains endroits dans le livre, il aurait été souhaitable que Chauvier se situe davantage par rapport aux travaux de ses contemporains. Par exemple, on aurait apprécié qu'au chapitre III l'auteur précise en quoi sa "position originelle globale» se distingue de celle présentée par Charles Beitz dans Political Theory of International Relations (Princeton University Press, 1979). Le lecteur regrettera aussi l'absence de référence aux travaux de David Held, auteur pourtant reconnu comme une autorité en matière de cosmopolitisme. Somme toute, l'ouvrage est convaincant, agréable à lire et empreint d'une rigueur analytique appréciable.

Cela étant dit, Justice internationale et solidarité déçoit dans ses conclusions. J'ai l'impression que l'auteur a déployé dans son étude un appareil conceptuel impressionnant pour finalement en arriver à un résultat quelque peu décevant. En effet, tout au long de son ouvrage, Chauvier critique la théorie du droit des gens de Rawls qu'il considère comme une simple théorie de la courtoisie internationale. L'approche privilégiée par Rawls, qui est centrée sur l'État et non sur l'individu, ne serait pas appropriée. Mais en bout de ligne, l'auteur se démarque-t-il vraiment de Rawls? Contrairement à ce que la critique aurait pu nous laisser croire, les 
propositions de Chauvier ne sont-elles pas équivalentes à celles de Rawls? De mon point vue, cela fait peu de doute: le principe de socialisation des avantages proposé par Chauvier à la fin de son ouvrage est tout à fait analogue au "devoir d'assistance» proposé par Rawls dans The Law of Peoples (Cambridge, Mass., Harvard University Press, 1999). Dans le cas contraire, à supposer que les propositions substantielles du philosophe français soient nettement novatrices par rapport à celles de Rawls, alors Chauvier devra nous expliquer pourquoi il s'est fait le défenseur des idées de Rawls dans un récent article ("Les principes de la justice distributive sont-ils applicables aux nations?", Revue de métaphysique et de morale, $\mathrm{n}^{\circ}$ 1, 2002, p. 123-143).

Ainsi se pourrait-il que cet ouvrage en fasse à la fois trop et pas assez, c'està-dire qu'il critiquerait trop pour le peu qu'il aurait finalement à proposer? Il reste que Justice internationale et solidarité pose d'importantes questions et qu'il initie le lecteur francophone aux débats les plus importants de l'heure en théorie de la justice internationale.

MARTIN LEBLANC Université de Montréal

\section{Julia Kristeva, Le Génie féminin. Hannah Arendt, Paris, Fayard, 1999, 408 pages.}

Linguiste, romancière, archéologue du langage, Julia Kristeva arpente depuis plus de trente ans les vastes palais du langage et l'univers symbolique humain sous différentes formes. On lui doit plusieurs ouvrages majeurs dans le domaine littéraire, sémiologique et linguistique, mais la grande originalité de l'œuvre de Kristeva est sans doute la contribution psychanalytique qu'elle apporte au domaine du langage et, ce faisant, la contribution qu'elle apporte au développement de la psychanalyse par ses analyses du langage. La folie, le langage, la révolte, l'amour et le féminin demeurent ses thèmes charnières. Ceci demeure vrai de son dernier ouvrage, une trilogie centrée sur le thème du "génie féminin ». Trois lieux définiront ce "génie du féminin»: la vie, la folie et les mots. Trois auteures correspondent à ces trois lieux, les "plus sensibles" du Xx siècle: Hannah Arendt, Mélanie Klein et Colette. Je présente ici le premier des tomes sur Hannah Arendt ainsi qu'un résumé de la trilogie telle que Kristeva l'annonce dans l'introduction de son livre.

D'un ton prophétique, Kristeva annonce une ère du féminin à venir: "le siècle prochain sera féminin pour le meilleur ou pour le pire" (p. 11). Le "génie", cette extraordinaire faculté qu'on accordait jadis à qui étaient bénis des dieux, s'est aujourd'hui mué en la capacité particulière à innover. On comprendra ainsi que la triologie commence avec Hannah Arendt, qui a fait de cette aptitude le centre de sa théorie de l'action. Le génie est celui qui sait innover; plus particulièrement, le génie caractérise la personne singulière dont les expériences propres, les "excès surprenants» de l'existence surgissent au-delà d'un univers de plus en plus standardisé (p. 8). Historiquement, les femmes semblent avoir été rejetées de cette catégorie, en vertu de leur don spécifique de maternité. L'expérience possible d'une "osmose avec l'espèce» différencie radicalement le genre féminin des hommes, mais cette différence justement s'ajoute aux nombreuses difficultés à manifester 